\title{
DIAGNÓSTICO EN EDUCACIÓN Y TRANSICIONES
}

\author{
EDUCATIONAL DIAGNOSIS AND TRANSITIONS
}

\author{
Ignacio Javier Alfaro Rocher* \\ Universidad de Valencia
}

\section{RESUMEN}

El artículo presenta una reflexión sobre el papel del "Diagnóstico en Educación" en las transiciones. Las premisas clásicas del diagnóstico tradicional se desmoronan con la incorporación de nuevas consideraciones relacionadas con el carácter procesual, la impredecibilidad del futuro y cambios sociales y con las nuevas tendencias metodológicas del diagnóstico. Desde una perspectiva sistémica, se sugiere un diagnóstico centrado en dos áreas prioritarias: a) la exploración de las causas que impiden el desarrollo de los individuos y el proceso de transformación de sus habilidades y, b) el compromiso paralelo con una transformación educativa y social. Asimismo, el artículo repasa el sentido de algunos conceptos relacionados con el diagnóstico: habilidades, empowerment, crisis, experimentación, personalidad, etc.

Palabras clave: Diagnóstico en Educación; habilidades, empowerment, crisis, experimentación personalidad.

\begin{abstract}
The article presents a reflection on the role of Educational Diagnosis in transitions. The classic premises of traditional diagnosis crumble with the incorporation of new considerations related with the procesual character, future's unpredictability and social changes, and with the new methodological tendencies of diagnosis. From a systemic perspective, it is suggested a diagnosis centered in two highpriority areas: a) the exploration of causes that hinder individuals' development and the process of transformation of their abilities and, b) the parallel commitment with an educational and social transformation. Likewise, the article reviews the sense of some concepts related with the diagnosis: abilities, empowerment, crisis, experimentation, personality, etc.
\end{abstract}

Key words: Educational Diagnosis; abilities; empowerment, crisis, experimentation; personality.

* Catedrático de Universidad de Diagnóstico en Educación. Departamento de Métodos de Investigación y Diagnóstico en Educación de la Universidad de Valencia. Avenida Blasco Ibáñez, 30. 46010-Valencia. (e-mail: ignacio.j.alfaro@uv.es). 


\section{Introducción}

El ser humano a lo largo de su ciclo vital va recorriendo diferentes espacios a los que se debe adaptar, va transitando por unos itinerarios de diverso tipo y recorriendo etapas cada vez más complejas, no exentas de crisis, y percibidas en múltiples ocasiones como procesos discontinuos. Lógicamente, el diagnóstico y la orientación deberían conocer mínimamente el proceso de desarrollo en cada una de las etapas con el fin de poder orientar, al menos, en los momentos decisivos por los que transitan las personas. Sin embargo, este comentario, que resulta tan evidente, podría estar cargado de múltiples prejuicios si se contempla desde concepciones clásicas de la orientación y del diagnóstico. Es el motivo principal de este artículo: analizar el papel del diagnóstico desde una perspectiva actual en el marco de las transiciones.

La literatura científica ha abordado en diversas ocasiones el tema de las transiciones, aunque de diferente forma en función del propio concepto de las mismas o de los enfoques teóricos y epistemológicos de cada cual. En primer lugar, realizaré algunas breves anotaciones generales acerca de este tema en el que subrayaré mi punto de vista conceptual y, seguidamente, efectuaré otras consideraciones acerca del papel del diagnóstico ante las transiciones.

\section{Habilidades y proyección temporal}

El tema de las transiciones va emparejado con la propia existencia humana así como con el progreso de la ciencia y de la sociedad. Sigue impregnando el pensamiento humano por su relación con el desconocido futuro, por su interpretación sobre el constante devenir y, en cierto modo, constituye una de las materias pendientes del diagnóstico y de la orientación. Precisamente, Robert W. Lent (2001: 222) señala que uno de los 12 objetivos de la psicología vocacional para la nueva década es "Incrementar nuestro conocimiento acerca de las diferentes transiciones laborales (p.e.: escuela-trabajo, trabajo-trabajo, desempleo-trabajo, trabajo-jubilación) y trasladar ese conocimiento al campo psicoeducativo y a otras intervenciones".

El enfoque teórico en el estudio de las transiciones ha sido variado y depende, entre otras cuestiones, de la condición profesional del analista, que en nuestro ámbito educativo enfatiza las variables relacionadas con el desarrollo del ser humano y con la educación. En este sentido, el ser humano va progresando inteligentemente y, a la par, va construyendo una sociedad cada vez más desarrollada y compleja, a la que las nuevas generaciones tienen que ir adaptándose, eliminando lo que estorba para el progreso y construyendo nuevos escenarios más desarrollados. Son procesos cíclicos, esquemas de aprendizaje, individual y colectivo, que tienen que ser experimentados, manipulados, automatizados y superados, con el fin de construir y alcanzar nuevas estructuras que mejoren la sociedad que nos hemos encontrado al nacer. Es decir, es el hombre -como entidad individual y social- quien siempre está presente como brazo impulsor de todas las transiciones y transformaciones hacia estados más evolucionados y, por lo general, los avances y cambios en los más diversos campos del conocimiento y de la sociedad siempre vienen del que ha llegado antes a niveles superiores de desarrollo y de inteligencia. 
En cualquier caso, existe una parcelación del conocimiento y una variedad de profesionales que analizan las diferentes transiciones; sin embargo, no podemos olvidar la interacción que se produce entre todas ellas. La siguiente tabla muestra diferentes formas de contemplar las transiciones, todas ellas interconectadas, aunque a nosotros nos corresponde intervenir preferentemente empezando por las primeras: evolutivas, académicas, profesionales, etc.

\begin{tabular}{|l|l|}
\hline TRANSICIONES & \multicolumn{1}{|c|}{ PATRONES DE CAMBIO EN: } \\
\hline Evolutivas & Sucesivas etapas del desarrollo a lo largo de todo el Ciclo Vital \\
\hline Académicas & A lo largo de la escolaridad, itinerarios formativos... \\
\hline Escuela-trabajo & Inserción laboral \\
\hline Profesionales & Dentro de una profesión y/o interprofesionales, desarrollo de la carrera.... \\
\hline Circunstanciales & $\begin{array}{l}\text { Aleatorio/vitales, desempleo, conyugales, fallecimiento allegados, } \\
\text { maternidad... }\end{array}$ \\
\hline Socio-históricas & $\begin{array}{l}\text { Generacionales, multiculturales, epidemias, guerras, migraciones, } \\
\text { pobreza... }\end{array}$ \\
\hline Económicas & $\begin{array}{l}\text { Globalización, economías informales, basadas en el conocimiento, } \\
\text { mercados,... }\end{array}$ \\
\hline Artísticas & Movimientos artísticos y culturales \\
\hline Políticas... & $\ldots$ \\
\hline
\end{tabular}

El diagnóstico y la orientación tienen siempre una tarea que es promover acciones educativas estratégicas para la ayuda a los individuos que transitan por esta sociedad y a lo largo de su vida. Éstas pueden ser individuales o grupales: acciones sobre el currículum, programas específicos de transición, asesoramiento individual y grupal, partenariado-coordinación, acciones políticas, etc. Sin embargo, las transiciones, una cuestión tan histórica como la vida misma, en muchas ocasiones han sido tratadas por la psicopedagogía desde puntos de vista, en mi opinión, no del todo educativos o centrados en el desarrollo de los individuos.

Los cambios y pluralidad de la sociedad actual ha hecho que se hable de interpretaciones holísticas de la realidad, de utilización de pluralidad de métodos, de perspectivas sistémicas, etc. El diagnóstico y la orientación se han lanzado a una interpretación global de los nuevos escenarios cambiantes en los cuales se produce una interacción emergente entre el contexto y el individuo, ambos indiscutiblemente en desarrollo. El desafio del diagnóstico es la explicación de la fenomenología explorada, individuo-contexto, desde una perspectiva sistémica, en constante evolución y progreso. Son cuestiones en cierto modo clásicas que recuerdan a filósofos como Heráclito ( $\pi \alpha ́ v \tau \alpha \rho \varepsilon \tilde{\imath})$ "No puede uno bañarse dos veces en el

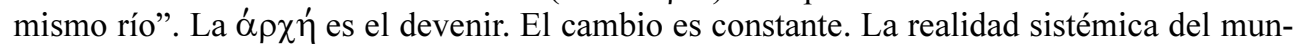
do, holística, está presidida por el azar y está abierta a acontecimientos creativos interactuantes. También se puede rememorar la dialéctica de Hegel, con su teoría de los contrarios y del fluir de las cosas, el eterno devenir no corre amorfo..., la dinámica evolutiva transforma el concepto en cuanto a contenido y a significado..., es un avance incesante hacia lo 
nuevo..., el avance dialéctico está inmerso en la naturaleza como una parte de un proceso universal, etc.

Algunas concepciones psicológicas o educativas actuales no han profundizado mucho más de lo que se entreve en estas notas. El diagnóstico se nutre, cada vez más, de una variedad metodológica, telemática, incluso estadística, mucho más compleja y elaborada que en otras épocas. A veces, perdido y dependiente de los avances tecnológicos actuales, sin una visión clara de la realidad educativa, de los aprendizajes, del cambio cualitativo, del desarrollo, etc. sin indagar en los procesos sistémicos de cambio y en los propios aprendizajes. Una cosa es utilizar una pluralidad metodológica para ver los problemas desde múltiples ópticas y, otra, optar por una explicación integrada, que incluya una interpretación unitaria e interactuante de la realidad; cuestión que muchos investigadores todavía no han llegado a entender. La interpretación unitaria se define psicológicamente dentro de en un proceso temporal dando sentido a los procesos de transformación de las habilidades.

Se ha demostrado (Secadas, 2000) que el factor temporal ejerce una influencia decisiva en la evolución de la inteligencia, en el desarrollo de los patrones cronológicos, históricos, culturales, etc. y en el proceso de desarrollo de cualquier habilidad. Del mismo modo, el factor espacial supone el inicio de un proceso de creación inteligente y, por ende, de cualquier estructura de habilidades. Sin embargo, difícilmente las estructuras de análisis se estancan en lo espacial, son sobre todo temporales y éste es el mayor reto del Diagnóstico y, probablemente, de la mente humana: la necesidad de atrapar en un momento psicológico los procesos efímeros, trabados en estructuras abarcables como algo unitario.

La difícil tarea del diagnóstico es, pues, interpretar grupos de habilidades que tengan sentido, como estructura dinámica, en un periodo de duración determinado y que, de alguna forma, sea posible percibirlas unitariamente.

En otras palabras, el análisis y diagnóstico de cualquier habilidad que no cuente con el factor temporal o con el desarrollo, carece de sentido psicológico y educativo. El tiempo es el organizador del desarrollo, de la conducta, la personalidad, la inteligencia y, en definitiva, de la propia existencia. Las habilidades a su vez, en su proyección temporal, dan sentido unitario a la conducta humana. Esta es la virtualidad de los nuevos enfoques metodológicos: poder entrar en el diagnóstico de procesos en construcción, dar sentido a la conducta y fomentar el crecimiento continuado del individuo o del grupo. No hay que olvidar que en este último se ponen en juego grupos de habilidades compartidas por sus elementos y que, en definitiva, son las que definen sistémicamente al grupo, a las organizaciones y a la institución educativa.

Según esto, el diagnóstico está siempre lidiando con transiciones y cambios, cuya esencia y sentido educativo hay que vislumbrar. Como decía antes, el matiz no es nada nuevo; sin embargo, previamente a cualquier tipo de análisis, se exige un posicionamiento claro sobre la óptica desde la que se prefiere actuar, que en el caso del diagnostico educativo y de la orientación debería ser el desarrollo del sujeto, eso sí, contemplado desde la pluralidad metodológica que se quiera. Pero, insisto, una interpretación global y sistémica no tiene por qué obscurecer el análisis del proceso creativo que nos traslada de un nivel a otro del desarrollo inteligente. 


\section{Clarificando las perspectivas de análisis en las transiciones}

En Orientación Profesional se ha definido la transición como un problema de socialización que discurre en paralelo a la continuidad de los cambios sociales y económicos cifrados en algunos aspectos, como son: el desempleo, los cambios de actitudes ante la educación y el trabajo, los cambios en las instituciones laborales, la globalización, el cambio en la economía, la estructura de trabajo, la variabilidad en el empleo, etc. El término de transición se adscribe a términos relacionados con crisis, transformación o cambio y, en definitiva, al

"abandono de un conjunto de asunciones previas y la adopción de otro conjunto nuevo que le permita afrontar un espacio vital alterado" (Rodríguez Moreno, 1998: 145 y ss.).

Esto es, un cambio en el contexto interno y otro en el externo.

En principio, hablar de transiciones implica asumir la existencia de fases, etapas, estadios o, sencillamente, de contextos de alguna forma diferenciados y cambiantes. Todo depende del marco de referencia desde donde se sitúe el análisis, en el sujeto o fuera del mismo. Lo cual indirectamente supone hablar inicialmente desde un punto de vista psicológico y evolutivo o desde una perspectiva sociológica, sin dejar de lado la perspectiva educativa.

Echeverría (1997) apunta dos grandes enfoques en el estudio de las transiciones,

“aparentemente irreconciliables: a) Psicoevolutivos: Enfatizan el proceso de construcción de la estructura individual de la personalidad y b) Sociológicos: Remarcan el proceso de incorporación a la sociedad." (op. cit., p. 88).

Declara que su opción aboga por una relación funcional en la que se conjuga un proceso de búsqueda de la identidad personal y una inserción socio-profesional (integración), en una relación dialéctica entre los procesos de: incorporación a la sociedad y de construcción de una estructura individual de la personalidad (op. cit., p. 89). El planteamiento es integrador, ya que como afirma más adelante,

"de poco sirven enfoques psicologicistas, que encorsetados en una función reflexiva sobre los determinantes personales acaban por responsabilizar casi exclusivamente al orientado del logro de los objetivos de cambio y desarrollo".

Del mismo modo, afirma que tampoco sirven los

"enfoques sociologicistas, que sólo centren su área de actuación en el cambio de los condicionamientos situacionales, a la espera de que éstos por sí solos generen la autorrealización de las personas" (op. cit., p. 101).

Se nos plantea, pues, un debate inicial que enfrenta la perspectiva psicológica-evolutiva y la sociológica. El citado autor aboga por una situación dialéctica integradora. En nuestra opinión es un debate de suma trascendencia por sus implicaciones en la investigación y en la práctica profesional. En cualquier caso, tampoco se puede en este corto espacio abordar algunos conceptos que van apareciendo en cada una de estas citas, por ejemplo, el relacionado con el significado de la construcción de una estructura de identidad personal con todos sus posibles matices. Sin embargo, continuaré presentando otras opiniones sobre las perspectivas de análisis en las transiciones. 
Gimeno Sacristán (1997, citado por Corominas e Isus (1998: 160-161) diferencia entre transiciones sincrónicas, diacrónicas y microtransiciones y las define en general de la siguiente manera

\begin{abstract}
"La transición es el momento y la experiencia de vivir la discontinuidad dentro de la natural multiplicidad, diversidad y polivalencia del mundo social y cultural en que nos toca vivir en cierto momento"... "A veces la frontera y la discontinuidad que provoca el hecho de que haya transiciones son la consecuencia que el propio sistema escolar sea resultado de una agregación de diversos tipos de educación..." (ibídem).
\end{abstract}

Según esta cita, se trata de un cambio producido en el contexto exterior (mundo social, cultural y sistema educativo) que el sujeto experimenta como una discontinuidad; es decir, en el exterior hay una discontinuidad y el sujeto la percibe.

En un sentido semejante, Schlossberg $(1984,1991)$ destaca por un lado el cambio producido en el exterior y, por otro, la percepción de la persona del cambio que se está produciendo fuera.

En ambos autores existe una coincidencia en que el cambio se produce en el mundo externo y, en consecuencia, el sujeto reacciona transformando la comprensión de sí mismo y del entorno. La transformación se produce fuera y, paralelamente, en la percepción del sujeto. En cierto modo, el cambio externo va por delante y le sigue la reacción del individuo que intenta acoplar su modo de pensar y su percepción a esos hechos y situaciones discontinuas. Es el entorno quien cambia y el individuo tiene que reaccionar al mismo. En cualquier caso, en mi opinión, no se da un énfasis suficiente a la posibilidad de una interacción sistémica que posibilite también la transformación de lo externo y no sólo de la vivencia y/o comprensión de sí mismo y del entorno.

Corominas e Isus (op. cit. p. 156 y 157) añaden que "las adaptaciones del individuo a las transiciones de la vida dependen básicamente de tres condiciones:

a) Percepción de la transición (según el esquema propuesto: normal o anormal, temporal o permanente, etc.),

b) Características del entorno previo y posterior a la transición, y

c) Características del individuo (rol de género, competencias psicosociales, etc.)”.

En este punto aparecen nuevos matices, se insiste en que se trata de una adaptación del individuo, aunque se añade algo más, que hay unas características previas del entorno y otras posteriores a la transición y que hay también unas características y competencias del sujeto. Entendemos que se amplía el punto de vista de las citas anteriores en el sentido de que se supone que el entorno no es estático o determinante, lo cual en cierto modo abre las esperanzas de no dejar al hombre como esclavo circunstancial de lo externo. Pero, en mi opinión, lo más importante es que, si se incluye esta perspectiva sistémica interactuante, se posibilita a la investigación y al diagnóstico para que, desde su condición autónoma y libre, pueda investigar no sólo las mejores estrategias adaptativas o el propio proceso interactivo que conjuga las competencias personales y el sistema educativo o laboral, sino también para evaluar si el sistema educativo en general es el mejor para el desarrollo integral (social, psicológico, etc.) de los sujetos o, por el contrario, debe cambiarse. El punto de vista de la investigación en las transiciones lo situamos sobre todo desde la perspectiva de la potenciación y fortalecimiento del propio sujeto y, por derivación, de 
su entorno. Es un compromiso que empieza por el sujeto y sigue por la activación de una intervención social.

En definitiva, una perspectiva diagnóstica y educativa en el tema de las transiciones tiene dos objetivos claros:

a) la adaptación e integración de los sujetos a las mismas y

b) la investigación acerca de si éstas son las más adecuadas para el enriquecimiento y desarrollo de los individuos.

En este sentido, si la siguiente cita se entiende de este modo, la subscribimos totalmente:

"En la línea del empowerment... la orientación no puede limitarse a una serie de técnicas sino que exige la implicación en la defensa de los derechos de los orientados y la participación en el proceso de mejora y transformación social" (Corominas e Isus, 1998, p. 182).

Así, volviendo al tema inicial, la perspectiva sociologicista se centra, en principio, en los cambios sociales, culturales y condicionamientos situacionales; sin embargo, no creemos que se preocupe por la incorporación del individuo a la sociedad. Ese interés es educativo así como la autorrealización de las personas. Lo que ocurre es que si se antepone erróneamente el cambio externo y se reduce la acción individual a una mera reacción adaptativa ante los acontecimientos, opino que no estamos hablando ni de perspectiva psicológica ni educativa. La perspectiva educativa estaría tras la búsqueda de una integración y realización del individuo en la sociedad y también de una transformación activa del entorno, si éste no es el más apropiado.

Por otra parte, la perspectiva psicologicista se ha definido como aquella que enfatiza la construcción de la estructura personal, como principal determinante del cambio y del desarrollo. Efectivamente, no podemos hablar de determinantes, pero sí de procesos interactivos de construcción y cambio. Nuestra matización insiste en que, desde una perspectiva auténticamente psicopedagógica, no es suficiente con localizar el cambio fuera y concebir al individuo como un ente que debe reaccionar adaptándose a los mismos, sino que la esencia del hombre es progresar, avanzar, desarrollarse y construir una transformación no sólo externa sino también en las propias personas y en su conducta inteligente. No es cuestión sólo de percepción de la realidad externa sino de transformación de sus competencias $y$ habilidades. Este es el reto del Diagnóstico en Educación, resaltar la importancia de las competencias y habilidades de los sujetos en la toma de decisiones; no dejar los procesos de aprendizaje y de construcción del conocimiento para los aprendizajes escolares básicos, como la lectura o escritura, y suponer que el resto de aprendizajes en la vida se rigen por otros cauces en los que no intervienen las habilidades previas, las competencias o ciertos planteamientos constructivistas. El diagnóstico no se reduce al análisis de los intereses, de las transiciones o a la recopilación de una abundante información acerca de los cambios y opciones en el proyecto vital. Hay algo más relacionado con el propio proceso de aprendizaje, con la formación recibida, con el desarrollo de competencias, con el ensayo y error, con la experimentación e incursión en experiencias de exploración profesional, con la investigación acerca de estos procesos de aprendizaje y de esta interacción del individuo con el entorno.

Recientemente, Donoso (2001), al hablar de las controversias para el avance en el diagnóstico en orientación profesional, se hacía la misma pregunta ¿por qué no intentar la 
aplicación de los planteamientos de la psicología vigostkiana de la zona de desarrollo próximo a la planificación y gestión de la carrera?

Esquematizando los amplios puntos de vista tenemos, por una parte, los enfoques centrados en la influencia de variables externas, entre los que destacan la perspectiva socioeconómica y la sociológica, y, por otra, los centrados en el individuo y en su formación con las perspectivas psicológica y educativa.

Así, desde el punto de vista externo, la perspectiva socioeconómica se ha interesado por la evolución del mercado de trabajo y sus efectos sobre la educación, la rentabilidad personal y social de la educación, la conectividad educación-empleo, etc. Su objetivo está más focalizado en la planificación, la acreditación, la evaluación de logros institucionales, etc. que en el diagnóstico aplicado. De forma semejante, la perspectiva sociológica se ha interesado en la clarificación del papel e influencia de ciertas variables como el género, clase social, el contexto socio-cultural, los perfiles generacionales o profesionales, fundamentalmente, con el fin de describir y comprender la lógica interna de las transiciones en función de tales variables.

Desde el punto de vista del sujeto, la perspectiva psicológica ha ido manejando variables relacionadas con la autoestima, el locus de control, aspectos emocionales, estrategias de afrontamiento, expectativas, intereses, autoconcepto, etc.; variables todas ellas importantes para el diagnóstico y la orientación, si existiera un determinismo evidente de las mismas que mediatizara en las transiciones. Finalmente, otras perspectivas educativas han estado interesadas en la planificación de los procesos de transición, en la valoración de necesidades, competencias, significados y creencias, en el establecimiento de programas de intervención, la gestión del proyecto profesional-vital, etc.

La aportación diagnóstica contempla los dos puntos de vista anteriores pero, desde su exploración y descripción proactiva, incide algo más en las variables que deben modificarse para fomentar el desarrollo personal de los sujetos, que normalmente se encuentran en el análisis profundo del propio proceso de transformación de habilidades y en el análisis acerca de si las condiciones externas son las más adecuadas para ese fortalecimiento personal o, por el contrario, se hace necesario una intervención paralela encaminada a provocar un cambio y transformación contextual que facilite el desarrollo de los individuos.

\section{Cambios en el Diagnóstico acordes con nuevas ideas sobre transiciones}

Una concepción clásica del diagnóstico se enfrenta profundamente con el concepto de transición. Tradicionalmente, se ha utilizado el diagnóstico para determinar el punto de partida de las habilidades, intereses, aptitudes, personalidad, conductas exploratorias, etc. de los individuos. Junto a ello, y supuestamente conocido el futuro que les depara, se trata de acoplar los dos sistemas sujeto-contexto de la mejor manera; es decir, de buscar la mejor adscripción en función de las destrezas y competencias exploradas en los sujetos. Esta concepción clásica del diagnóstico no es muy lejana. De hecho, en la actualidad se han ido perfeccionando y actualizando los instrumentos diagnósticos así como su complejidad, condiciones y tipo de administración, etc.; pero sin embargo, en muchos de los casos, se espera del diagnóstico lo mismo que en otras épocas, un ajuste entre las características del 
individuo y las del predecible entorno, cuestión que choca con dos elementos imperantes en este momento y opuestos a tales premisas: el carácter procesual del diagnóstico (apartado 3.1) y la impredecibilidad del futuro (apartado 3.2); por no citar también, la desconexión de estas estrategias clásicas con las nuevas tendencias metodológicas del diagnóstico (apartado 3.3).

\section{Una apuesta decidida por el proceso}

El diagnóstico hace tiempo que ha abandonado la estrategia de hacer descripciones de constructos estáticos derivados de ciertos enfoques psicométricos. Inevitablemente, la historia de los métodos de evaluación y de diagnóstico ha estado unida a los grandes movimientos teóricos psicológicos y pedagógicos. El diagnóstico prescriptivo tuvo su contexto en la medición de la inteligencia y en los tests de aptitudes, la teoría de rasgos y factores, el propio uso de los tests en diagnóstico con la constante utilización de pruebas de selección, tests estandarizados para la evaluación de destrezas, intereses, etc. Esta aproximación diagnóstica, basada en la descripción psicométrica del sujeto y en sus potencialidades afortunadamente parece que ha tocado fondo.

Actualmente, y a pesar de las limitaciones de los tests clásicos como instrumentos de medida en el proceso diagnóstico, existe un campo importante en la medición y evaluación educativa centrado en la elaboración de pruebas estandarizadas referidas a dominios, tests adaptativos informatizados, etc. Son procedimientos útiles para informar sobre las ejecuciones de los estudiantes, representan muestras perfectamente elaboradas de sus dominios; sin embargo, desde nuestro punto de vista, dan muy poca información relevante para la toma de decisiones en diagnóstico. En realidad, ocurre como con los tests psicométricos anteriores, no son pruebas diagnósticas: aquellos los denominábamos en otro lugar descripciones psicológicas de los sujetos, éstos últimos son sólo descripciones evaluativas sobre el estado alcanzado en materia de dominios y destrezas. Ambas aproximaciones adolecen del clásico sentido procesual del diagnóstico. Realizar una descripción de una "estructura mental" no significa haber llegado al proceso, habida cuenta también del carácter cambiante y progresivo de las estructuras cognitivas a lo largo del desarrollo. Los resultados de los tests son una muestra más de una conducta o parcela determinada en un momento determinado de un proceso. El diagnóstico es algo más amplio y el concepto de pruebas diagnósticas también. Los nuevos "Standards for Educational and Psychological Testing" (AERA, APA y NCME, 1999), incluyen en su consideración no sólo los tests para la evaluación psicológica en sentido clásico, sino también otras técnicas y estrategias de exploración: el performance assessment, el portfolio, etc. que informan más acerca del proceso y de las estrategias que se van utilizando paulatinamente. Estas técnicas se acercan más al conocimiento de las habilidades que dispone el sujeto para ir escalando los diferentes niveles de dificultad para la realización de una tarea, cuestión que simula más un proceso de ejecución y, a la postre, una transición entre diferentes niveles de habilidad.

Por otra parte, existe asimismo otras investigaciones básicas que han puesto en tela de juicio el sustrato teórico en el que se mantenía el diagnóstico basado en descripciones de constructos aptitudinales más o menos estáticos, y a otros temas sobre la estabilidad, modificabilidad y heredabilidad de las aptitudes, todos ellos tratados en otro lugar (Alfaro, 2001a). Lo importante ahora es resaltar que el diagnóstico no puede quedarse en la búsque- 
da de un acoplamiento entre dos supuestos constructos estáticos -el individuo y el contexto-, cuando en realidad se trata de dos elementos cambiantes, dinámicos y en constante transformación.

El reto del Diagnóstico en Educación indudablemente está en insertarse en el propio proceso de transformación de habilidades, en la construcción de una identidad personal y profesional y en la inserción en los procesos aprendizaje relacionados con un proyecto de vida. El prefijo "dia-" ( $\delta i \alpha ́)$ tiene dos acepciones: "a través de" y "a lo largo de". En la actualidad se está recuperando precisamente el discurso de la segunda acepción, es decir, la indagación "a lo largo del proceso", la búsqueda de "lo que concurre, de lo que va a la par" de un proceso, que es precisamente el auténtico significado del término "síndromos" ( $\sigma u ́ v \delta \eta \circ \mu \varsigma)$, tan unido a la historia del diagnóstico.

\section{Impredecibilidad del futuro y cambio}

Otro elemento que atenta contra el mencionado ajuste "individuo-contexto" está relacionado con el constante cambio y transformación que está sufriendo la sociedad actual. Se habla de globalización de la economía, de transformación de la estructura de trabajo, de sociedades multiculturales, de acceso creciente de la mujer al trabajo, de nuevas tecnologías, de economías informales y emergentes, etc.

Recientemente, Marín y Rodríguez (2001: 316-317) en un trabajo sobre "prospectiva del diagnóstico y la orientación" han resaltado el carácter impredecible del futuro y de las transformaciones estructurales que está sufriendo nuestra sociedad. Se suman a la opinión de Geissler, quien afirma que si queremos preguntarnos por el futuro, no tenemos que acudir a los políticos, adivinos, obispos o padres, sino que debemos preguntarnos a nosotros mismos y después navegar por internet. (ibidem).

"Será el aprendizaje (la educación) el que nos dé la competencia para tal menester"

Añaden, asimismo, que los profesionales de la orientación han de proyectar las consecuencias deseables y no deseables para intentar, a través de múltiples estrategias de acción, que las segundas aminoren su impacto y que, de esta manera, sea patente el futuro deseable y que el orientado intervenga activamente en la consecución del futuro deseado.

Dos consecuencias se extraen de estos comentarios: una relacionada con el aprendizaje y la educación y otra con el papel facilitador del orientador, en cuanto intenta reducir la distancia entre el futuro probable y el futuro preferible. Añadiría algo más, considero que mayor será la ayuda en esta reducción si el diagnóstico se centra preferentemente en ese conocimiento de uno mismo y en ese proceso de aprendizaje. Es una forma de construir el presente y empezar a vivir el futuro.

Todas las acciones humanas tienen una finalidad proactiva, una teleología, se hacen para algo, incluso la sana costumbre de aburrirse. Parece obvio que la simple revisión e indagación del presente debería tener alguna proyección futura. Pero nunca viviremos en el futuro, sino en la prolongación presente de nuestra existencia, de la que fragüemos de hoy a entonces. El futuro no es algo utópico o lejano, se crea día a día. No tendría sentido dejar que el diagnóstico se redujera a la simple valoración del estado de las cosas o al estableci- 
miento de criterios, sin una influencia en el futuro, sin una constante creación interactiva de estructuras personales o sociales más optimizadas. Así pues, la aspiración, tanto del diagnóstico como de la orientación, no es sólo la toma de decisiones sino la creación de ese futuro mejor, aportando claves ante lo desconocido, que tengan en cuenta las habilidades, aspiraciones y comportamientos presentes (psíquicos, morales, conductuales...) así como las necesidades y habilidades colectivas, y que se proyecten hacia objetivos proporcionales a la preparación y desarrollo alcanzados.

Básicamente, la finalidad última de ambas disciplinas pasa por ese proceso de análisis y de compromiso investigador aplicado tanto a las habilidades y progresión en los aprendizajes como al establecimiento de unas condiciones estructurales que faciliten ese desarrollo individual y social. Para ello, indudablemente, es necesario la participación activa de todos los agentes involucrados en el proceso educativo y el convencimiento de que una intervención en el desarrollo individual de los individuos es también un elemento transformador y de progreso de nuestra sociedad.

Algunas de estas ideas se reflejan en el cambio que se está produciendo en algunas orientaciones teóricas, por ejemplo, el concepto de carrera defendido por Watts ya no está ligado a la clásica transición del mundo educativo al laboral y luego al ascenso dentro de este.

"El concepto de carrera debe llegar a definirse no de forma objetiva como hasta ahora, sino de forma subjetiva para describir la progresión de una persona a través del aprendizaje $y$ del trabajo, concebido como un proceso que puede seguir un curso tanto horizontal como vertical, en el campo del trabajo y del aprendizaje, y dentro y fuera de las organizaciones"... Se trata de buscar "un sistema de cualificación y acreditación que evalúe e informe sobre el aprendizaje de las personas no sólo dentro del sistema educativo sino fuera de él". En esta línea Watss (1996) señala que algunos países están desarrollando un sistema integrado de cualificaciones profesionales que cubran diferentes formas de aprendizaje”. (Marín y Rodríguez, 2001: 319).

La impredecibilidad del futuro se alivia apostando por el desarrollo del aprendizaje en el presente, con el fortalecimiento (empowerment) de las personas, con el desarrollo de competencias en los nuevos dominios de aprendizaje. El diagnóstico se sitúa en el establecimiento del nivel de habilidades previo del cual parte el sujeto y en la búsqueda de los obstáculos que impiden un progreso adecuado en sucesivos niveles de habilidad o en determinadas transiciones que, fundamentalmente, son el cambio y progreso a nuevas estructuras de habilidad sobre la base de las ya automatizadas.

En definitiva, cuando se habla de aprendizaje, necesariamente se está incidiendo en el propio proceso de desarrollo de la inteligencia, entendida en un sentido amplio. Hay que conocer, valorar y fomentar habilidades inteligentes, las apropiadas para cada momento evolutivo, de forma que los estudiantes tengan los suficientes recursos y destrezas para construir su propio presente y futuro. El punto de mira de esta matización obliga a investigar y avivar el desarrollo personal en todos sus frentes y, consecuentemente, se espera que este enriquecimiento provoque paulatinamente los cambios en el contexto $\mathrm{y}$, en definitiva, en la sociedad. 


\section{Nuevas tendencias metodológicas}

Otro reto que tiene el diagnóstico es incorporar de una manera decidida las nuevas tendencias metodológicas que se están incorporando en diversos ámbitos educativos, y que, en general, podrían denominarse como evaluación alternativa o auténtica, y que dentro de este movimiento aparecen con diversas denominaciones: alternative assessment, performance assessment, portfolio, authentic assessment, etc.

El denominador común de todas ellas es que se requiere que los estudiantes, en vez de escoger respuestas como en los tests clásicos, las generen, centrando la evaluación en ejecuciones y tareas reales y contextualizadas; lo que supone la incorporación de estrategias de análisis sobre cómo los estudiantes planifican, organizan y ejecutan las tareas, sus disposiciones y habilidades metacognitvas, sus destrezas, etc.

En concreto, el performance assessment intenta emular el contexto o condiciones de la vida real dónde se aplican de hecho los conocimientos y habilidades específicas. La ejecución de las tareas propuestas en estos tests a menudo requieren períodos de tiempo relativamente amplios, cuyo rango va desde unos pocos minutos hasta una clase o más de varias horas o días. Ejemplos de tales performances pueden incluir resolución de problemas usando materiales, haciendo inferencias complejas después de recoger información o explicando oralmente o por escrito la razón de ser de un desarrollo particular de una actuación gubernamental bajo unas condiciones económicas dadas. Se entiende que en esta evaluación se incluye el rendimiento individual así como el de un equipo de estudiantes (AERA et alt. 1999: 137).

Los nuevos estándares citados consideran las "performance assessments" como tests que difieren de los clásicos en que los evaluados tienen que demostrar sus habilidades ejecutándolas en contextos semejantes a la vida real.

"Por ejemplo, la evaluación de un psicólogo en prácticas puede requerir que éste entreviste a un cliente, escoja tests apropiados y llegue a un diagnóstico y a un plan para la terapia" (op. cit., p. 41).

Así estas pruebas son de diversa naturaleza, pueden estar basadas en productos como en conductas, etc. de forma que sus resultados deberían poder ser generalizables a amplios dominios. Por ello, es necesario que estos tests incluyan especificaciones, dimensiones críticas evaluables, criterios de puntuación, definición del dominio, etc. Asimismo,

"cuando las pruebas están diseñadas para elicitar procesos cognitivos complejos, el análisis lógico de las tareas y el análisis empírico y lógico de la ejecución del tomador del test en las tareas necesariamente debe proporcionar evidencias de validez" (op. cit. p. 42).

Los retos de este tipo de evaluación se localizan en la estandarización, generalización, administración y puntuaciones obtenidas, por ejemplo, en el sentido de que se requiere un incremento de la fiabilidad y exactitud de los juicios, debido a complejidad de los procedimientos de evaluación, las puntuaciones, los materiales, tiempos, etc. Sin embargo, las posibilidades diagnósticas son inmensas ya que se ofrece al sujeto una manera de adentrarse en la práctica y de ejecutar una tarea que implica la puesta en marcha de un conjunto de habilidades $y$, por ende, el establecimiento de niveles diagnósticos en diferentes partes de proceso. 
El portafolio es otra metodología que los mismos estándares la entienden como un tipo de performance assessment Así, los portafolios (scorables) son recolecciones sistemáticas de productos típicos recogidos a lo largo de un período y con la posibilidad de modificación sobre la marcha. El propósito específico es incluir productos representativos del estudiante o indicadores de progreso. El reto también se fija en la comparabilidad de las puntuaciones, en los procedimientos de administración, etc. (AERA et alt. 1999).

En general, estas metodologías suponen un enfoque evaluativo dinámico que enfatiza las aplicaciones y se centra en la evaluación de la conducta directa utilizando problemas realistas y promoviendo el pensamiento aplicado a problemas abiertos y poco definidos. El portafolio incluye una variedad de contextos, métodos, propósitos, etc. Los sujetos pueden participar en su diseño, en la evaluación de materiales, pueden trabajar juntos, evaluador y evaluado, en la selección de materiales, etc. Todos estos aspectos deben constar en las especificaciones del portafolio. (Rodríguez Espinar, 1997; Alfaro 1998; AERA et. alt., 1999; Alfaro 2001).

La variedad de estrategias metodológicas emergentes es considerable. Por ejemplo, en los estudios de psicología de la Universidad de North Carolina-Chapel Hill. El portafolio se va desarrollando acumulativamente durante los tres primeros años y es evaluado por el profesorado al final de cada año. La evaluación no tiene efectos sancionadores de (aprobar o suspender) sino que su objeto es orientar el desarrollo de la carrera futura del estudiante. El portafolio consta de aspectos relacionados con diferentes núcleos: plan de estudios incluyendo intereses de investigación, metas profesionales y plan para lograrlo, cursos recibidos y por recibir, currículum, experiencia con ordenadores, en investigación y artículos publicados o publicables. Al final de cada año los estudiantes reciben un informe escrito acerca de su evolución en estos puntos, junto con una lista de sugerencias acerca de cómo debe evolucionar el desarrollo del portafolio en el futuro.

En el ámbito del desarrollo de la carrera se han elaborado guías con estándares o competencias y pautas-guía para la autorreflexión: autoconocimiento, planificación y exploración de carreras, roles de la vida, desarrollo educativo, etc. El reto es ofrecer a los estudiantes la oportunidad de conocer los principales elementos sobre los que pueden ser evaluados. (Lester y Perry, 1995). Rodríguez Espinar (1997: 191) presenta un modelo de portafolios desarrollado conjuntamente por la Asociación Americana de Orientadores Escolares y del Comité Nacional de Coordinación Ocupacional, cuyo título es "Get a Life: Your Personal Planning Portfolio for Career Development". El objetivo del portafolio, por una parte, "facilita al alumno los materiales necesarios para recabar información para el conocimiento de sí mismo y el desarrollo de su carrera así como para hacer un uso correcto de la misma; por otra, facilita a orientadores y profesores la estructura y conceptualización que hace posible la sinergias entre contenido y procedimientos".

La estructura del portafolios se compone de cuatro secciones:

a) autoconocimiento (autoconcepto, habilidades interpersonales, personales...);

b) los diferentes roles en la vida (aceptación de mí mismo, influencia familiar, mi tiempo libre ...);

c) Desarrollo educativo (mi estilo de aprendizaje, mis habilidades, mi rendimiento, mi potencias, mi trabajo escolar...); y 
d) Exploración y planificación profesional (conocimiento del mundo del trabajo, información profesional, mis experiencias de exploración...).

Cada sección está dividida en dos partes: una ficha personal, destinada a recoger información y reflexiones sobre el contenido de la sección, y una ficha de competencias, que recoge el nivel de logro de las diferentes competencias de la sección, así como las actividades y experiencias educativo-profesionales que se han realizado. (Rodríguez, 1997).

Corominas (2000) incide también en el portafolio como recurso para el desarrollo profesional en la orientación psicopedagógica y comenta diferentes modelos: Career Portfolios (http://www.stw.ed. gov/Databases/Subject2), Get a Life; Career Options Planner and Career Options Portfolio, y Like Work Portfolio. En resumen, el artículo ofrece un panorama amplio acerca de su uso tanto como guía en el proceso formativo de adquisición de competencias profesionales, así como elemento para conseguir trabajo y para mantenerse y progresar en el mundo laboral. Lo importante es que

"el portafolio en la orientación profesional es la herramienta que permite organizar la información sobre sí mismo y utilizarla en las decisiones personales educativas y profesionales. Al mimo tiempo, introduce la idea de que se trata de un proceso que continúa a lo largo de la vida y que la persona ha de ser un negociador de su carrera profesional, siendo el responsable de su propio desarrollo" (Corominas, 2000: 516).

Evidentemente, la utilización de algunas de estas estrategias no invalida el uso diagnóstico de algunos tests estandarizados; de hecho, en los portafolios suelen guardarse documentos significativos relacionados con tests aplicados, etc.

Como se deduce de estas dos nuevas tendencias metodológicas comentadas lo importante es que se cambian los parámetros del sistema de evaluación; por ello, se incluyen dentro de lo que también se llama enfoques dinámicos y evaluación auténtica (authentic assessment).

La authentic assessment es una evaluación alejada de instrumentos artificiales que, por una parte, supone una toma de decisiones basada sobre la comprensión completa, global, significativa y real de los procesos de aprendizaje y de su significado y, por otra, representa una visión holística de su progreso, de la construcción de sus aprendizajes, de sus experiencias en el desarrollo de los mismos e incluso de las actividades e interacciones con el profesor u otros compañeros. La evaluación auténtica considera, pues, personas, familias, tareas y ambientes. (Alfaro, 1998).

En síntesis, los enfoques dinámicos denuncian que el enfoque tradicional del diagnóstico mide sólo el aprendizaje como acumulación de conocimientos, no tiene en cuenta el proceso o se refiere a normas. En contraposición, estos enfoque pretenden integrar la evaluación (y no sólo el test) en el sistema de aprendizaje y de enseñanza y aumentar la validez de la evaluación intentando medir lo que el estudiante hace. Para que se denomine dinámico, se supone que el proceso de evaluación debe promover el aprendizaje. Por ello, medición auténtica y medición cognitiva se solapan hasta cierto punto, pero no completamente. En la medida en que la evaluación tenga valor en sí misma, ésta se considera incluida dentro de la evaluación auténtica. Según esta perspectiva muchos sistemas de evaluación cognitivos no están considerados como medición auténtica, particularmente, aquellos que efectúan una medición indirecta del rendimiento o de sus habilidades. 
En general, se puede considerar que la medición auténtica evalúa productos acabados o conductas en acción, en este último caso con frecuencia la evaluación es naturalista, y se utilizan procedimientos documentales para seguir la conducta del estudiante.

La evaluación auténtica supone, pues, que el individuo genera, al ser evaluado, una actividad relacionada con la construcción del conocimiento, con el propio corpus disciplinar, pero sobre todo, conecta con los problemas del mundo más allá de la escuela (Wiggins, 1998, pp. 25-28).

En cualquier caso, este enfoque dinámico, al igual que ocurría con la medición de aptitudes, considera la evaluación más en su función diagnóstica y centrada en el proceso y progreso de los estudiantes que en la clasificación o escalación de personas. No es de extrañar que el enfoque tradicional haya estado más ligado a la tecnología psicométrica, centrándose consecuentemente en la selección y en la validez predictiva.

\section{Diagnóstico, habilidades y experimentación}

He propuesto en sucesivas ocasiones un concepto de diagnóstico incardinado en el aprendizaje y desarrollo de los individuos. Defino el diagnóstico como

"el proceso de búsqueda de las causas que impiden el progreso desde niveles de desarrollo de una habilidad determinada, individual o colectiva, hasta la creación de nuevas estructuras hábiles de nivel superior y su posterior automatización, con el fin de optimizar dicha transición dentro del propio proceso educativo" (Alfaro, 2001a: 177).

Es una conceptuación aplicable a cualquier progreso y transición a lo largo del ciclo vital. Las transiciones implican la transformación de unas estructuras previas de habilidad en otras más avanzadas acordes con las nuevas exigencias de desarrollo de la persona en el contexto vital en el que se desenvuelve. El diagnóstico, por consiguiente, debe incidir en el mismo proceso de transformación de la conducta inteligente, en las causas que pueden impedir esa transición de unas habilidades a otras.

Algunos pueden concebir la inteligencia exclusivamente como una capacidad general de adaptarse a cualquier situación impuesta desde fuera; el cambio se produce fuera y luego el individuo reacciona, se transforma como consecuencia de ese cambio.

El contenido de la ciencia psicopedagógica no puede quedarse en contemplar cómo se reacciona ante los cambios sociales, ese es el contenido de otras ciencias afines. Si se hace esto, no hay una ciencia que radique en el individuo y se esclaviza a éste en función de los sistemas educativos o sociales imperantes que, como indica la educación comparada, son diversos a lo largo del mundo y no todos perfectos. En concreto, los cambios en las estructuras docentes y en el sistema educativo deberían estar ocasionados porque previamente se ha investigado el funcionamiento del individuo y porque su desarrollo demanda que se adopte un sistema educativo acorde con este desarrollo ¿Por qué existen diferencias en los planteamientos de la diversidad y excepcionalidad entre los diversos sistemas educativos? ¿De qué criterios dependen las políticas educativas en materia de inserción profesional o empleo? ¿El papel del profesional se reduce al acomodo de los individuos a los sistemas?, etc.

Como decíamos, hay un postulado que entiende que el individuo posee una capacidad de adaptación inteligente y que ésta tiene que producirse de acuerdo con las características 
de lo cambiado. Se le impone al individuo un sistema pero no se le tiene en cuenta para nada. La investigación universitaria y aplicada, en mi opinión, está en la obligación de estudiar los cambios y transformaciones que operan en el individuo en diferentes áreas de su comportamiento y proponer sistemas educativos y estrategias que ayuden a su crecimiento psicológico, intelectual, cultural y profesional. Es decir, es el individuo quien exige unos cambios; mientras que los intereses del sistema podrían ir perfectamente por otros derroteros, aunque se espera que no.

Antes, al mencionar del concepto de Diagnóstico en Educación nos situábamos al lado del proceso de crecimiento de los sujetos. Este proceso está en múltiples áreas y, desde este punto de vista, las perspectivas de investigación son amplias y variadas. Nosotros en algunos trabajos hemos visto que existen cambios significativos en la estructura de habilidad de los sujetos, por ejemplo, en torno a los cinco años existe un salto significativo del signo al símbolo, que se está produciendo en múltiples aprendizajes, por ejemplo, en la escritura, la lectura y las matemáticas (Secadas, Rodríguez y Alfaro, 1994; Alfaro, 2001a). Así, por estas edades se define un tránsito en el funcionamiento cognitivo que contextualiza la propia estructura de habilidades y destrezas. Se está produciendo una transformación sumamente interesante para el diagnóstico. En consecuencia con nuestra aportación, esta estructura psicológica reclama unos sistemas de enseñanza-aprendizaje acordes con su nivel de competencias. No al revés, como se deduce de otros planteamientos, es decir, propuesto un sistema, que básicamente no responde a criterios de desarrollo evolutivo sino a otros externos, buscar cómo adaptamos el individuo al sistema.

Por ello, valorando también la importancia de cualquier otra actividad investigadora, reclamamos un espacio en el diagnóstico que se ocupe del desarrollo de los diversos aprendizajes que se suceden cíclicamente sobre la base de estructuras de habilidad determinadas. Y estos aprendizajes están en múltiples ámbitos: el escolar, el profesional, el relacional, laboral, familiar, etc.

El criterio de las transiciones lo estamos fijando en competencias, habilidades, cambios y procesos de transformación en los individuos, que reclaman a su vez cambios en los sistemas y, en donde, en buena lógica, existen interacciones sistémicas con el medio y un feedback constructivo. Del mismo modo, también valoramos una actividad profesional importante tendente a facilitar un proceso de adaptación a los sistemas, siempre que no se olvide que éstos están al servicio de un conjunto de características individuales y de desarrollo personal.

En este sentido, son buenos los programas educativos y estrategias profesionales que ayudan a facilitar el tránsito a lo largo de los sistemas educativos combinando la información, la reflexión, el autoconocimiento y la práctica gradual de los objetivos próximos de desarrollo y de crecimiento. Esencialmente, es la estructura superior de habilidad quien reclama la reorganización y flexibilización de las estructuras precedentes y, para ello, es importante facilitar la construcción y el progreso, situarse al lado del sujeto, proponer actividades en las que se pueda poner a prueba y ejercitar los sucesivos niveles de habilidad más complejos. En la propia manipulación y ejercitación de tales actividades exploratorias se sitúa preferentemente la cognición y el diagnóstico. No es cuestión de aflorar a la consciencia las vivencias y percepciones acerca de la discontinuidad y de las transiciones, como si validáramos el supuesto aforismo socrático de quien conoce su bien (ó $\gamma \alpha \theta$ óv) y su virtud 


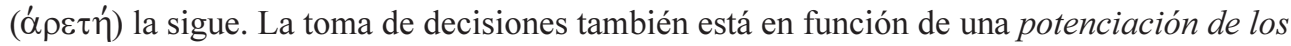
recursos y de las habilidades de las personas.

Así pues, la primacía psicológica no está en la situación o en el cambio externo sino en las condiciones para resolver los dilemas, en las habilidades oportunas para adaptarse a la nueva situación. Cada vez más, adquieren mayor relevancia el constructo competencias profesionales, las dimensiones de conocimientos de aprendizaje, de formación, unido todo ello a otras dimensiones personales, relacionales, interpersonales, de trabajo en equipo, etc. El reto del diagnóstico es entrar en esas dimensiones y establecer el nivel de logro en cada una de ellas, sobre la base de la experimentación del sujeto en situaciones simuladas o reales. El avance en el aprendizaje requiere una experimentación en situaciones nuevas, un ensayo y error, una nueva combinación de habilidades tendente a la solución de nuevos problemas, un ensayo de transición de unas habilidades a otras. Todo ello, supone la inserción del diagnóstico y de la orientación en ese proceso de construcción inteligente del proyecto de vida y de las competencias necesarias para desenvolverse adecuadamente en un mundo cambiante.

El desafío del diagnóstico en orientación profesional, por ejemplo, más que dedicarse al ajuste paternalista individuo-contexto antes comentado, es conseguir adentrarse en el currículum y en los propios procesos de construcción de competencias profesionales. Es otro aprendizaje objeto del diagnóstico, como pudiera ser la lectoescritura en los niveles inferiores del sistema educativo. Son procesos de aprendizaje. La única diferencia es que ahora se trata de competencias profesionales. Competencias que son entendidas como una combinación de cualificación adquirida en la formación técnica y profesional, el comportamiento social, la capacidad para trabajar en equipo, la capacidad de iniciativa y la predisposición al riesgo (Delors, 1996).

Existen numerosas experiencias de inserción en las que, desde un punto de vista psicológico, se experimenta y se pone a prueba la estructura de habilidades de los sujetos. Experimentación proviene de "empíria", al igual que pirata, $\pi \varepsilon 1 \rho \alpha \tau \eta$ s, el que experimenta, explora, etc. [ $\pi \varepsilon \imath \rho \alpha ́ \omega \omega=$ emprender, ensayar, explorar]. La inteligencia humana necesita del ensayo y exploración de estructuras nuevas para avanzar, no basta con los procesos de concienciación o metacognición, que incluso pueden romper los automatismos consolidados necesarios para el avance. Orientar es facilitar esta toma de contacto con nuevas situaciones; es ayudar a capacitar al individuo para que pueda afrontar sus transiciones; es facilitar una formación y exploración continua, en donde se promuevan sus competencias de manera que el diagnóstico pueda insertarse en tales procesos de aprendizaje.

Lo importante es poner al sujeto en experimentación, de tal forma que sea la habilidad superior, la que reclame la reorganización de las precedentes. Probablemente, así consigamos sucesivas transiciones en términos de habilidad. Es la única forma psicológica de avanzar en el desarrollo humano. La toma de decisiones es patrimonio exclusivo de cada persona y depende, además de otras cuestiones circunstanciales a veces determinantes, del nivel de autoconocimiento que tiene cada individuo acerca de su nivel de competencias y de sus expectativas de desarrollo. Otro tipo de asesoramiento, más protector o basado en juicios fortuitos, puede invadir el terreno de lo privado y podría significar una falta de confianza o invasión en la autonomía decisoria de cada persona. El individuo debe sentir, y sufrir, esa autonomía en la toma de decisiones $\mathrm{y}$, a ser posible, sobre la base de una experimentación previa. 
El diagnóstico, por una parte, debe observar las habilidades que están en juego en los aprendizajes, más que otros aspectos relacionados con factores psicométricos subyacentes a los aprendizajes y, por otra, debe estar pendiente de esa reorganización de habilidades, ya sedimentadas, dominadas o automatizadas que intentan conseguir nuevas estructuras habilidosas, en las que el individuo ensaya, con lo ya dominado, nuevas formas, combinaciones o mecanismos para alcanzar esa nueva estructura de habilidad. Es en ese ensayo donde hay que considerar una buena parte de los errores, básicamente como intentos frustrados de conseguir una nueva habilidad. En términos diagnósticos, los errores son la pista ineludible que detecta, por una parte, la habilidad que poseen los sujetos y, por otra, la estructura que pretenden conseguir. (Alfaro, 2001b).

\section{Crisis, progreso y personalidad}

De lo expuesto podemos diferenciar dos ámbitos de cambio:

a) los que acontecen en los sistemas (educativos, sociales, económicos, etc.), que hay que evaluar constantemente, y

b) los observados en el desarrollo personal de los sujetos.

En relación con los primeros los cambios obedecen a múltiples factores y a su combinación, por ejemplo, factores educativos, políticos, económicos, sociales, administrativos, geográficos, etc.

En relación a los segundos, los cambios y transiciones de los sujetos, se podría afirmar que el hecho de hablar de transiciones en sentido clásico es asumir que la transformación y el crecimiento no es constante y progresivo. Es como si lo precedente no valiese para lo nuevo. Sin embargo, esto no es así. Todo en el individuo está cambiando constantemente y, a la vez, se pueden establecer momentos distintos por una mayor densidad de ciertas operaciones y de ciertos estados y condicionamientos, incluso sociales, económicos, familiares, culturales, etc. Hay unas operaciones mentales y características de conducta por las que se puede definir que objetivamente un individuo ha sufrido una transición, su estilo de operar es de otra manera. No se puede negar las transiciones y la psicología evolutiva ha dado suficiente muestra de ello.

Desde una perspectiva diagnóstica el quid de la cuestión está en definir el proceso de las transiciones a partir del desarrollo de los sujetos y, a partir de ahí, realizar propuestas psicopedagógicas en las situaciones escolares para que el sujeto siga realizando sus transiciones y sus cambios.

En este constante proceso de transformación se puede establecer unos períodos o etapas. Su determinación dependerá lógicamente de las unidades de análisis, del contenido observado o de las propias adscripciones teóricas de los proponentes. En cualquier caso, siempre se manifiestan unas transiciones entre unos estadios y otros. Echeverría (1997) afirma que

"cada uno de los estadios se relaciona con todos los demás y de su correcta interrelación depende el desarrollo adecuado de la secuencia. Por otra parte, cada estadio existe de alguna forma antes de que "su" periodo decisivo y crítico llegue normalmente" (op. cit., p. 93).

Efectivamente, es innegable la presencia en determinados momentos de un tipo de operaciones o comportamientos y del surgimiento de otros diferentes ante nuevos desafíos del 
desarrollo. Estos cambios no están exentos de períodos de crisis. En este sentido, el mencionado autor comparte el criterio de Erikson relacionado con este fenómeno:

"El término crisis se usa aquí en un sentido evolutivo para connotar no una amenaza o catástrofe, sino un momento decisivo, un periodo crucial de vulnerabilidad incrementada y potencial y, por lo tanto, fuente ontogenética de fuerza y desajuste generacional" (Erikson, 1971, citado por Echeverría, op. cit. p. 93).

En nuestra opinión, las crisis no son amenazas de nada sino periodos normales en los que determinadas estructuras y habilidades son inservibles y necesitan combinarse y flexibilizar para alcanzar nuevos progresos. De hecho, el término crisis implica, por una parte, una mutación importante en el desarrollo de los procesos (крıбí) y por otra, su raíz pro-

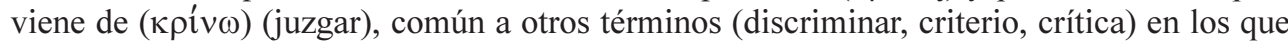
se debe juzgar y tomar decisiones en función de criterios, que habitualmente están relacionados con el nivel competencial de la persona en el cual se encuentra cada uno más cómodo. El periodo crítico en las transiciones es aquel en el que se abandona el hábito anterior por insuficiente y se flexibiliza para elaborar el siguiente y ese período también puede ser crítico, conflictivo, dificil, de estudio y de esfuerzo.

En cada crisis se está poniendo a prueba una estructura competencial diferente a otras anteriores; son elementos necesarios para el avance en los que se está reorganizando una estructura cohesiva de habilidades, inteligencia, personalidad, intereses, motivaciones, etc. y en donde el individuo, con mayor o menor dilación temporal, pone todo su empeño emocional para solucionar el reto de progreso. En este sentido, habrá que ir dejando de lado en el campo del Diagnóstico en Educación una concepción patológica de las crisis para incidir, por el contrario, en facilitar esos tránsitos necesarios, lo que indirectamente supone contemplar la prevención desde una óptica que signifique preparar y disponer de los elementos necesarios para que se realicen satisfactoriamente estos cambios, en vez de contemplarla como la eliminación o reducción de riesgos de una forma proteccionista. Lo importante es que el sujeto cada vez tenga mayores recursos, capacidades y competencias vitales.

En las crisis, en realidad, el sujeto está flexibilizando la rutina que se ha introducido en el aprendizaje en general. Los hábitos aprendidos de una determinada manera se hacen incompatibles y friccionan con nuevos avances. Los cambios son necesarios para ajustarse a la nueva estructura que se está creando con experiencias de todo tipo y de una forma coherente con toda su personalidad. Por ello, hemos defendido que el diagnóstico debe acompañar a los sujetos en todos los aprendizajes a lo largo del ciclo vital. No puede acudir solamente cuando ocurran situaciones de crisis radical o traumática; sería no sólo asumir un diagnóstico de tipo remedial sino también olvidar que para la toma de decisiones es necesario que el sujeto esté dotado de recursos para solucionarlas y, habrá que decir que, si se ha llegado a un punto crítico y traumático de ruptura, es precisamente porque se ha producido un abandono en la tarea educativa.

Por otra parte, también es cierto que cuando el individuo se encuentra ante problemas de cambio radical en los sistemas externos que desconoce y no tiene experiencia en ese cambio, es importante que se realice una orientación y que se estimule una elección basada en una serie de habilidades y condicionantes dirigidos a un proyecto de realización personal. 
"Orientar en el proceso de transición es una tarea holística, que abarca la persona en todas sus facetas. Para ello, más que ayudar, aconsejar o asistir, consiste en mediar, poner en contacto, los aspectos personales con la nueva situación. Orientar significa capacitar a las personas para que tomen decisiones adecuadas" (Corominas e Isus, 1998, p. 158).

El equipo formado por Echeverría, Isús, Sarasola, Figuera, etc. (Echeverría, 1997) ha desarrollado un modelo holístico de orientación para determinados momentos y transiciones, en el que se combina el conocimiento de las oportunidades existentes y de uno mismo, los determinantes sociales y personales, el proyecto de vida y las necesidades.

En definitiva, en cada transición el individuo realiza una doble acción que Echeverría lo sintetiza en dos procesos:

“-Incorporación a la sociedad, que supone la vivencia de un rol reconocido...

- Construcción de una estructura individual de la personalidad...” (Echeverría, 1997, p. 89).

Personalmente, comparto esta doble función aunque matizando que se debería integrar ambas acciones expresándolo en el sentido de "desarrollar su personalidad mediante la integración en el nuevo rol". Sencillamente por evitar el peligro expuesto antes relacionado con anteponer la situación externa al conjunto de variables que definen la personalidad de un individuo y, también, porque en esa incorporación experiencial, en esa puesta en contacto con habilidades de otro nivel, está la construcción de la personalidad.

Así, es plenamente lícito resaltar el papel que juega la personalidad en la toma de decisiones ante la incorporación en un rol o en un proyecto que incluye un cierto tipo de habilidades y, todavía más, si se tiene en cuenta que la personalidad es el conjunto de habilidades que una persona ha ido creando a lo largo de su vida. Las habilidades son esas formas de soporte de la personalidad en funcionamiento. A lo largo del desarrollo se van sucediendo diferentes niveles de comportamiento hábil, que definen a las personas diferencialmente, pero que forman una estructura cohesiva en la que se incluye no sólo la inteligencia, sino el afecto, la personalidad, etc. El comportamiento es único, integrando todos estos aspectos diferenciales que dan una visión de conjunto de cada persona en progresivos momentos de desarrollo. El diagnóstico puede acercarse a este proceso a través de diferentes formas de observación y de análisis. Se trata de analizar sus habilidades y ejecuciones, la forma de proceder y, consecuentemente, interpretar el sentido psicológico de las mismas. (Alfaro, 2001a).

La intervención orientadora debe ayudar a descubrir ese entramado cohesivo de las propias capacidades y habilidades, que conforman la personalidad, así como las exigencias y oportunidades del entorno. En este sentido, uno de los enfoques alternativos a los modelos tradicionales sobre personalidad -la teoría multifactorial sistémica de Royce y Powell- coincide con esta interpretación unitaria de la personalidad, destacando precisamente esa inclusión en la organización de la personalidad de aspectos cognitivos, aptitudes y afectivos:

"Los procesos afectivos han constituido el punto focal de muchos análisis teóricos de la personalidad, pero es realmente la combinación de cognición y afecto lo que constituye el corazón del funcionamiento integrado humano y de las diferencias individuales en la conducta, pensamiento, emoción, arousal, etc." (Royce y Powell, citados por Sánchez Cánovas y Sánchez López, 1999, p. 217). 
Nuestro acercamiento, sin embargo, insiste preferentemente en el análisis de las habilidades que se ponen en juego en cada uno de los aprendizajes, más que en definir una estructura de las aptitudes jerárquicamente organizada. La estructura cognitiva va evolucionando cualitativamente a lo largo del desarrollo y las habilidades se van depositando directa y paulatinamente en las tareas y aprendizajes, en la experimentación y no en el pensamiento (Secadas, 2000; Alfaro, 2001a).

En las transiciones se trata, pues, de formar a la persona para que sea capaz de abordar los nuevos retos, valorando los aspectos personales y potencialidades, la información selectiva que se disponga y facilitando las estrategias oportunas para que pueda flexibilizar los hábitos previos y acomodarlos a las demandas de desarrollo de un proyecto personal.

\section{A modo de conclusión}

Las diferentes perspectivas de análisis de las transiciones enfatizan con mayor o menor intensidad o bien el contexto interno del sujeto (personalidad, intereses...) o el externo (los cambios sociales, educativos...), dependiendo de sus respectivos planteamientos teóricos. En principio, la perspectiva diagnóstica está interesada en el desarrollo y construcción personal de los individuos en cada una de sus facetas vitales. Sin embargo, habría que hacer alguna matización, por ejemplo, que existe un proceso de transformación y de desarrollo personal que sigue unas pautas más o menos comunes para cada uno de los grupos de edad a lo largo del desarrollo y otras que responden a los cambios en el mundo externo dependientes de momentos históricos, sociales, educativos o culturales, y que interactúan con las anteriores. Se define, pues, un escenario de constante evolución y progreso en el que interactúan sistémicamente variables intrapersonales e intraculturales.

El Diagnóstico en Educación se diferencia de otras perspectivas por su interés en investigar las causas que impiden una transición progresiva a niveles de habilidad más complejos e inteligentes; por su interés en conseguir un enriquecimiento y desarrollo de los individuos y por su incidencia en la provocación de cambios en contextos externos cuando éstos no sean los más apropiados para el progreso de los individuos e, indirectamente, de la sociedad. En este sentido, el diagnóstico también está interesado en las transiciones y cambios externos, por cuanto que éstos pueden provocar conflictos en los sujetos al poner en juego diferentes estructuras de habilidad, a veces incompatibles con las nuevas situaciones que se presentan. Significa esto que el diagnóstico no puede desatender tampoco una exigencia de cambio y un compromiso con la adaptación de las políticas económicas, educativas y sociales a los intereses de desarrollo de los individuos y grupos sociales.

En cualquier caso, desde la perspectiva que se defiende en este trabajo, el eje vertebrador del diagnóstico es la resolución de los conflictos en las transiciones, a través del fortalecimiento y potenciación de los individuos en estrategias y competencias que incidan en la automatización de las precedentes, de forma que puedan enfrentarse con éxito a los retos de las nuevas situaciones cambiantes. Así pues, las estrategias de análisis diagnóstico se concentran básicamente en la exploración del estado de habilidades y competencias en cada uno de los periodos de aprendizaje a lo largo del ciclo vital adentrándose en los procesos de cambio que obligan a una reestructuración y flexibilización de habilidades previas tendentes a la consecución de nuevos desarrollos. 


\section{Referencias bibliográficas}

A.E.R.A., A.P.A. y N.C.M.E. (1999). Standards for Educational and Psychological Testing. Washington: American Educational Research Association.

Alfaro, I. (1998). "Tendencias en al Evaluación psicopedagógica: un acercamiento a través del ámbito de las dificultades de aprendizaje”. Revista de Investigación Educativa, 16 (2), 125-154.

Alfaro, I. (2001a). Proyecto Docente y de Investigación. Concurso-Oposición Cátedra de Universidad: Diagnóstico en Educación. Valencia: Facultad de Filosofía y Ciencias de la Educación (Inédito).

Alfaro, I. (2001b). "El tratamiento de los errores en el Diagnóstico en Educación”. Revista de Investigación Educativa, 19 (2), 433-452.

Corominas, E. (2000). “¿Entramos en la era portafolio?”. Bordón, 52 (4), 509-521.

Corominas, E. e Isús, S. (1998). "Transiciones y Orientación”. Revista de Investigación Educativa, 16 (2), 155-184.

Delors, J. (Coord.) (1996). La educación encierra un tesoro. Madrid: Santillana/Unesco.

Donoso, T. (2001). "Diagnóstico en Orientación Profesional: Controversias para el avance". Revista de Investigación Educativa, 19 (2), 453-458.

Echeverría, B. (1997). "Inserción sociolaboral”. Revista de Investigación Educativa, 15 (2), 85-115.

Lester, J. N. \& Perry, N.S. (1995). "Assessing Career Development with Portfolios". ERIC Digests: ED391110.

Lent, R. W. (2001). "Vocational Psychology and Career Counseling: Inventing the Future". Journal of Vocational Behavior, 59, 213-225.

Marín, M. A, y Rodríguez Espinar, S. (2001). "Prospectiva del diagnóstico y la Orientación". Revista de Investigación Educativa, 19 (2), 315-362.

Rodríguez Espinar, S. (1997). "El portafolios: ¿Modelo de evaluación o simple historial del alumno?”. En H. Salmerón (Coord.) Diagnosticar en Educación (pp. 183-199) Granada: FETE-UGT.

Sánchez Cánovas, J. y Sánchez López, M. P. (1999). Psicología de la diversidad humana. Madrid: Editorial Centro de estudios Ramón Areces, S.A.

Schlossberg, N. K. (1984). Counseling adults in transition, linking practice with theory. New York: Springer $\mathrm{Pb}$. Co.

Schlossberg, N. K. (1991). "A model for analyzing human adaptation to transition". The Counseling Psychologist, 9 (2), 2-18.

Secadas, F.; Rodríguez, M. T. y Alfaro, I. (1994). Escribir es fácil. Madrid: TEA Ediciones S.A.

Secadas, F. (2000). Del juego a la inteligencia. Psicología y trascendencia educativa del juego. Madrid: Autor.

Wiggins, G. P. (1998). Educative Assessment: Design Assessments to Inform and Improve Student Performance. San Francisco: Jossey-Bass Publishers.

Fecha de recepción: 07-10-03

Fecha de revisión: 05-04-03

Fecha de aceptación: 12-05-04 